\title{
Calibration Using Matrix Completion With Application to Ultrasound Tomography
}

\author{
Reza Parhizkar, Member, IEEE, Amin Karbasi, Member, IEEE, Sewoong Oh, Member, IEEE, and \\ Martin Vetterli, Fellow, IEEE
}

\begin{abstract}
We study the application of matrix completion in the process of calibrating physical devices. In particular we propose an algorithm together with reconstruction bounds for calibrating circular ultrasound tomography devices. We use the time-of-flight (ToF) measurements between sensor pairs in a homogeneous medium to calibrate the system. The calibration process consists of a low-rank matrix completion algorithm to de-noise and estimate random and structured missing ToFs, and the classic multi-dimensional scaling method to estimate the sensor positions from the ToF measurements. We provide theoretical bounds on the calibration error. Several simulations are conducted to evaluate the theoretical results presented in this paper.
\end{abstract}

Index Terms-Calibration, matrix completion, multidimensional scaling, sensor localization, ultrasound tomography.

\section{INTRODUCTION}

I $\mathrm{N}$ most applications involving sensing, finding the correct positions of the sensors is of crucial importance for obtaining reliable results. This is particularly true in the case of inverse problems which can be very sensitive to incorrect sensor placement. This requirement can be satisfied in two ways; We might put the effort in the construction of the instruments and try to place the sensors exactly in the desired positions, or use a method to find the exact positions after the construction of the device. In this work we consider the latter and we call the procedure of obtaining the sensor positions calibration. Note that even in the former case, due to the limited precision of the construction instruments, a calibration is needed afterwards to determine the exact sensor positions.

This work focuses on the calibration problem in circular ultrasound tomography devices, in particular, the ones manufactured and deployed in [2], [3]. These devices consist of a circular

Manuscript received December 21, 2012; revised April 24, 2013; accepted June 12, 2013. Date of publication July 11, 2013; date of current version September 04, 2013. The associate editor coordinating the review of this manuscript and approving it for publication was Dr. Yufei Huang. This work was supported by the Swiss National Science Foundation under Grant 200021-121935 and by Support for Frontier Research-SPARSAMERC Advanced under Grant 247006. This work appeared in part in the IEEE International Conference on Acoustics, Speech, and Signal Processing, Prague, Czech Republic, May 2011, pp. 549-552.

R. Parhizkar and M. Vetterli are with the School of Computer and Communication Sciences, Ecole Polytechnique Fédérale de Lausanne (EPFL), CH-1015 Lausanne, Switzerland (e-mails: reza.parhizkar@epfl.ch; martin.vetterli@epfl. ch).

A. Karbasi is with the Computer Science Department, ETHZ, Universitätstrasse 6, CH-8092 Zurich, Switzerland (e-mail: amin.karbasi@inf.ethz.ch).

$\mathrm{S}$. Oh is with the Industrial and Enterprise Systems Engineering Department, University of Illinois at Urbana-Champaign, Urbana, IL 61801 USA (e-mail: swoh@illinois.edu).

Digital Object Identifier 10.1109/TSP.2013.2272925 ring surrounding an object and scanning horizontal planes. U1trasound sensors are placed on the interior boundary of the ring and act both as transmitters and receivers. In such tomography devices, the sensors are not exactly placed on a perfect circle. This uncertainty in the positions of the sensors acts as a source of error in the reconstruction algorithms that are used to obtain the characteristics of the enclosed object. We aim at finding a simple method for calibrating the system at low cost and without using any extra calibrating instrument.

We use the time-of-flight (ToF) between each pair of sensors to estimate their mutual distances. In a homogeneous medium where the sound speed is fixed, the ToFs between sensors have a simple relation to their mutual distances. If we have all the noiseless ToF measurements, we can use the classic multi-dimensional scaling (MDS) method [4] to estimate the sensor positions. In practical settings however, there are a number of challenges; these measurements are noisy, not all the measurements are available, and there is an unknown delay added to the ToF measurements. We categorize the missing measurements into two classes; structured missing entries caused by inability of the sensors to compute the ToF with their close-by neighbors, and random missing entries due to malfunctioning of the sensors and uncertainties of the measurement procedure.

\section{A. Contributions}

In general, it is a difficult task to infer missing entries of a matrix. However, it has recently been established that if the matrix is low rank, a small random subset of its entries permits an exact reconstruction [5]. We use an interesting property of $\mathrm{Eu}-$ clidean distance matrices to show that a modified version of the ToF matrix (composed of ToFs between pairs of sensors) is low rank. Thus, its missing entries can be accurately estimated using matrix completion algorithms. We use OptSpace, a robust matrix completion algorithm developed by Keshavan et al. [6] to estimate the missing entries of this matrix. After completing the ToF matrix, we use a classic MDS algorithm (MDSLocalize) [4] to estimate the position of the sensors.

Besides providing a complete framework for self-calibration of circular ultrasound tomography devices, one of the main contributions of this work is the theoretical bound on the calibration error. Such results, as we see in the next section, are not valid only for ultrasound tomography devices, but also benefit the analysis of sensor localization algorithms for which local connectivity information is missing.

We also present a heuristic algorithm to estimate the unknown time delay, that is due to the unknown piezoelectric impulse response and lack of the time-origin in the measurements. The 


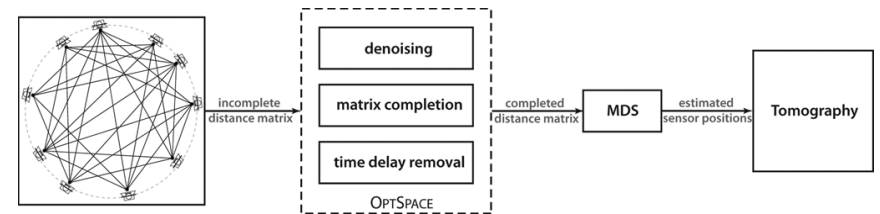

Fig. 1. Block diagram for the calibration procedure prior to ultrasound tomography. The incomplete distance matrix is passed through the OptSpace algorithm which denoises it, estimates the missing entries and removes the unknown time delay. The calibration is finished then by applying the MDS algorithm on the completed matrix which estimates the actual sensor positions.

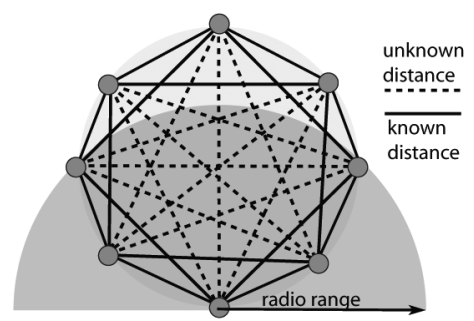

(a)

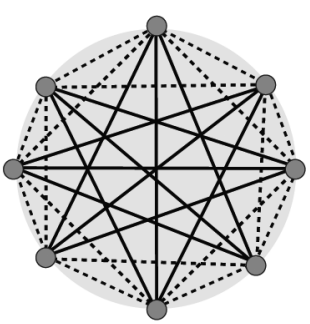

(b)
Fig. 2. Calibration with missing entries (a) in sensor localization local connectivity information is available. (b) in calibration the opposite is true.

block diagram shown in Fig. 1 summarizes the calibration procedure taking place prior to tomography.

\section{B. Related Work}

Calibration for circular tomography devices is a variant of sensor localization, a problem that has been extensively studied for the past decade [7], [8]. In sensor localization, given the local connectivity, the objective is to infer the global position of the sensors [9]-[11]. In practice, each node in the sensor network has a small communication range compared to the field size they are installed. This situation is depicted in Fig. 2(a).

In our problem, however, the local connectivity is precisely the kind of information that is missing (see Section II -B). This situation is demonstrated in Fig. 2(b). By comparing these two scenarios, one can think of the calibration problem for ultrasound sensors as the dual problem of sensor localization. As a result, all sensor localization algorithms that rely on local information/connectivity are doomed to fail in our scenario. To confirm this fact, in Section VI through numerical simulations we compare the performance of our proposed method with the state-of-the-art algorithms for sensor localization applied in our setting.

The first sensor localization algorithm we consider is Mds-Map[12]. In this method the distance of sensors that are not in each others vicinity is approximated by the shortest path between them. We can easily see that given the distances of faraway sensors, the shortest path is a very coarse estimate of the distance between the close-by sensors. This makes Mds-Map perform poorly in our setting. Further, we compare our results to one of the most prominent algorithms for centralized sensor localization, based on semi-definite programming (Sdp). The main problem of Sdp-based methods is their heavy computational load. According to [13], the sensor localization for more than 200 sensors is computationally prohibitive. Theoretical guarantees of such methods were provided recently by Javanmard et al. [14]. As their results suggest, once the number of sensors grows, we cannot reduce the error of semidefinite programming below a threshold unless we increase the communication range. We show, however, using the matrix completion, the error decreases as the number of transmitters/receivers grows.

In the core of our proposed method is matrix completion. It is easy to show that a matrix formed by pairwise distances is low rank (see Lemma 1). Based on this property, Drineas et al. suggest using matrix completion for inferring the unknown distances [4]. However, their analysis relies on the assumption that even for faraway nodes, there is a nonzero probability of communication. In our setting, this assumption implies that the pairwise distances of nearby transmitters/receivers can be obtained with a nonzero probability, which does not hold.

In a series of papers [6], [15], [16], Keshavan et al. study an efficient implementation of a matrix completion algorithm called OptSpace. We show that OptSpace is also capable of finding the missing nearby distances in our scenario and hence provide us with their corresponding ToFs. To the best of our knowledge, all the above work, as well as the recent matrix completion algorithms [17], [18], only deal with the random missing entries. However, in our case, we have structured missing entries in addition to random ones (see Section II-B), an aspect that was absent from the previous work. Therefore, one of our contributions is to provide analytic bounds on the error of OptSpace in the presence of structured missing entries.

The organization of this paper is as follows; In Section II, we define the model used in circular tomography and introduce the tools for calibration in such a setup. In Section III, we present the mathematical basis for the problem. Then in Section IV our main results for calibration are presented. Section V contains the proofs for the main results and finally Section VI is devoted to the simulation results.

\section{Circular Time of Flight TOMOgRaphy}

The focus of this research is ultrasound tomography with circular apertures. In this setup, $n$ ultrasound transmitters and receivers are installed on the interior edge of a circular ring and an object with unknown acoustic characteristics is placed inside the ring. The general configuration for such a tomography device is depicted in Fig. 3. At each time instance a transmitter is fired, sending ultrasound signals with frequencies ranging from hundreds to thousands of $\mathrm{kHz}$, while the rest of the sensors record the received signals. The same process is repeated for all the transmitters. Each one of $n$ sensors on the ring is capable of transmitting and receiving ultrasound signals. By employing these measurements, an inverse problem is constructed, whose solution provides the acoustic characteristics of the enclosed object (e.g., sound speed, sound attenuation, etc.). In order to solve the inverse problem, a very precise estimate of the sensor positions is needed. In most applications (e.g., [19], [20]) it is assumed that the sensors are positioned equidistant apart on a circle and no later calibration is performed to find the exact sensor positions.

\section{A. Homogeneous Medium and Dimensionality Reduction}

Assume that the mutual ToFs are stored in a matrix $\boldsymbol{T}$. In a homogeneous medium, entries of $\boldsymbol{T}$ represent the time travelled 


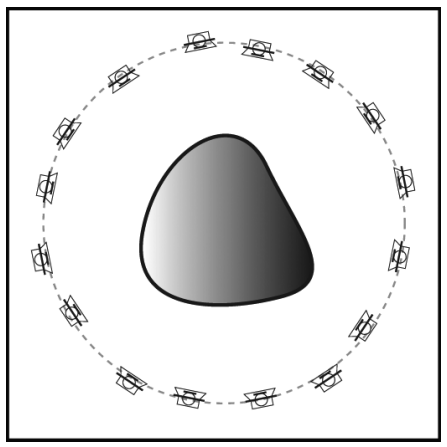

Fig. 3. Circular setup for ultrasound tomography considered in this work. Ultrasound transducers are distributed on the edge of a circular ring and the object with unknown characteristics is put inside.

by sound in a straight line between each pair of a transmitter and receiver.

Knowing the temperature and the characteristics of the medium inside the ring, we can accurately estimate the constant sound speed $c_{0}$. Thus, it is reasonable to assume that $c_{0}$ is fixed and known. We can construct a distance matrix $\boldsymbol{D}$ consisting of the mutual distances between the sensors as

$$
\boldsymbol{D}=\left[d_{i, j}\right]=c_{0} \boldsymbol{T}, \quad \boldsymbol{T}=\left[t_{i, j}\right], \quad i, j \in\{1, \ldots, n\}
$$

where $t_{i, j}$ is the ToF between sensors $i$ and $j$ and $n$ is the total number of sensors around the circular ring. Notice that the only difference between the ToF matrix $\boldsymbol{T}$, and distance matrix $\boldsymbol{D}$, is the constant $c_{0}$. Thus in the following, we focus mainly on the distance matrix rather than the actual measured matrix $\boldsymbol{T}$.

The matrix $\boldsymbol{T}$ is a symmetric matrix with zeros on the diagonal and so is the matrix $\boldsymbol{D}$. Although the distance matrix $\boldsymbol{D}$ is full rank in general, a simple element-wise transform of its entries leads to a low rank matrix. More precisely, we can prove (see Appendix A) the following lemma:

Lemma 1: The squared distance matrix

$$
\overline{\boldsymbol{D}}=\left[d_{i, j}^{2}\right],
$$

has rank at most 4 [4] and if the sensors are placed on a circle, its rank is exactly 3 .

\section{B. Time-of-Flight Estimation}

Several methods for ToF estimation (also known as timedelay estimation in acoustics [21]) are proposed in the signal processing community [22], [23]. Normally the received signal is compared to a reference signal (ideally the sent signal), and the relative delay between the two signals is estimated. As the sent signal is not available in most cases, the received signal through the object is compared to the received signal when the underlying medium is homogeneous. This assumption is not true in our case. In the calibration phase, we have only signals passed though the homogeneous medium. Thus, there is not any reference signal to find the relative time-of-flights.

Because of the above limitations, we are forced to estimate the absolute ToFs. For this purpose, we use the first arrival to estimate the ToFs.

In practical screening systems, to record measurements for one fired transmitter, all the sensors are turned on simultaneously and after some unknown transition time (which is caused

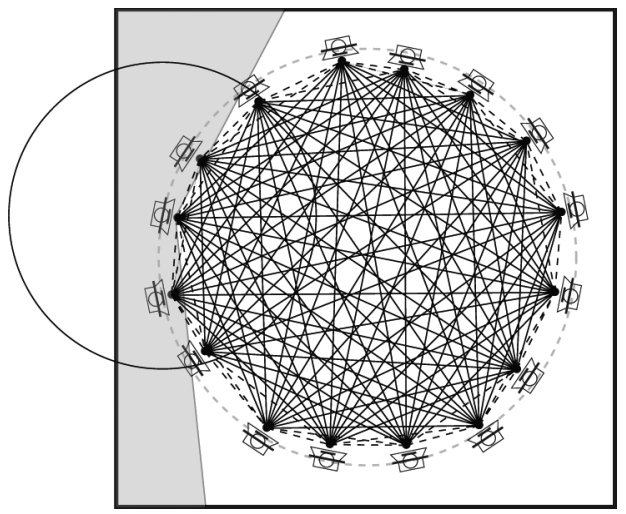

Fig. 4. The beam width of the transmitter causes the neighbouring sensors to not have reliable ToF measurements. This is shown by dashed lines and results in the structured missing entries.

by the system structure, different sensor responses, etc.), the transmitter is fed with the electrical signal and the receivers start recording the signal. This unknown time may change for each pair of transmitters and receivers. We will see that this unknown time delay plays an important role in calibration.

The beam width of the transducers and the transition behaviour of the ultrasonic sensors prevent the sensors to have a reliable ToF measurement for close-by neighbours. This causes incorrect ToF values for the sensors positioned close to each other. Therefore, numbering the sensors on the ring by their angles from 1 to $n$, in the ToF matrix $\boldsymbol{T}$, there are no measurements on a certain band around the main diagonal and on the lower left and upper right parts as well. We call these missing entries as structured missing entries. This is illustrated in Fig. 4. The links shown by dashed lines do not contribute to the ToF measurements, because the beam for the transmitter does not cover the gray part.

Compared to other measuring sensors such as X-ray and electromagnetic imaging devices, ultrasound sensors are more prone to malfunctions due to continuous contact with the measurement liquid (water, gel or oil). Further, since these devices are inside liquid, a momentary presence of an air bubble can cause an error in the sensor measurements. Studies also highlight the possibility of malfunction of ultrasound sensors [24]. Thus, during the measurement procedure, it might happen that some sensors produce outliers. One can perform a post processing on the measurements, in which a smoothness criterion is defined and the measurements not satisfying this criterion are removed from the ToF matrix. We address these entries as random missing entries. An instance of the ToF matrix with the structured and random effects is shown in Fig. 5, where $\boldsymbol{T}_{\text {inc }}$ denotes the incomplete ToF matrix and the gray entries correspond to the missing entries. Furthermore, in practice, the measurements are corrupted by noise.

Thus, we have an incomplete and noisy matrix $\boldsymbol{T}$, which cannot be used for position reconstruction, unless the time delay effect is removed, the unknown entries are estimated, and the noise is smoothed.

\section{Problem Setting}

For simplicity, we will assume that the unknown time delay is constant for all the transmitters, namely all the transmitters 


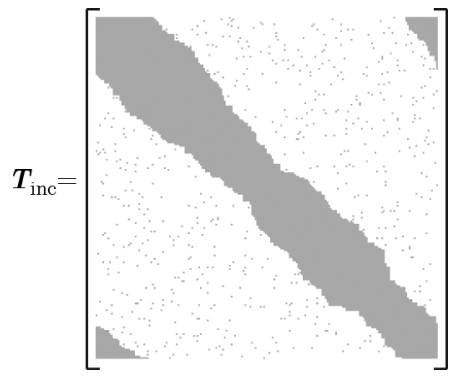

Fig. 5. A sample incomplete ToF matrix with structured and random missing entries.

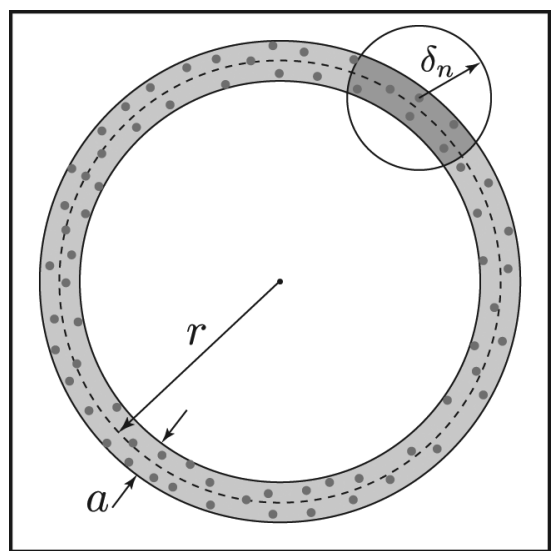

Fig. 6. Sensors are distributed around a circle of radius $r$ with small deviations from the circumference.

send the electrical signal after some fixed but unknown delay $t_{0}$. Hence, we can rewrite the distance matrix as

$$
\widetilde{\boldsymbol{D}}=\boldsymbol{D}+d_{0} \boldsymbol{A}+\boldsymbol{Z}
$$

where $\boldsymbol{D}=c_{0} \boldsymbol{T}, d_{0}=c_{0} t_{0}, \boldsymbol{Z}=c_{0} \boldsymbol{Z}_{0}$ and

$$
\boldsymbol{A}=\left[a_{i, j}\right], \quad a_{i, j}= \begin{cases}1 & \text { if } i \neq j, \\ 0 & \text { otherwise. }\end{cases}
$$

The matrix $\boldsymbol{Z}_{0}$ models the noise in the ToF measurements. In practice, the sensor positions deviate from the circumference of a ring and our ultimate goal is to estimate these deviations or equivalently the correct positions (see Fig. 6). The general positions taken by sensors are denoted by the set of vectors $\left\{\boldsymbol{x}_{1}, \ldots, \boldsymbol{x}_{n}\right\}$.

As described earlier, there are two types of missing entries; structured and random. Structured and random missing entries. To model the structured missing entries, we assume that any measurements between sensors of distance less than $\delta_{n}$ are missing (see Fig. 6). Hence, the number of structured missing entries depends on $\delta_{n}^{2}$. We are interested in the regime where we have a small number of structured missing entries per row in the large systems limit. Accordingly, a typical range for $\delta_{n}$ is $\delta_{n}=\Theta(r \sqrt{\log n / n})$. A random set of structured missing indices $S \subseteq[n] \times[n]$ is defined from $\left\{\boldsymbol{x}_{i}\right\}$ and $\delta_{n}$, by

$$
S=\left\{(i, j): d_{i, j} \leq \delta_{n} \text { and } i \neq j\right\},
$$

where $d_{i, j}=\left\|\boldsymbol{x}_{i}-\boldsymbol{x}_{j}\right\|$. Then, the structured missing entries are denoted by a matrix

$$
\boldsymbol{D}_{i, j}^{s}= \begin{cases}\boldsymbol{D}_{i, j} & \text { if }(i, j) \in S, \\ 0 & \text { otherwise. }\end{cases}
$$

Note that the matrix $\boldsymbol{D}^{\bar{s}}=\boldsymbol{D}-\boldsymbol{D}^{s}$ captures the noiseless distance measurements that is not effected by structured missing entries. This way, we can interpret the matrix $D^{s}$ as additive noise in our model. Likewise, for the constant additive time delay we can define

$$
\boldsymbol{A}_{i, j}^{\bar{s}}= \begin{cases}\boldsymbol{A}_{i, j} & \text { if }(i, j) \in S^{\perp}, \\ 0 & \text { otherwise }\end{cases}
$$

where $S^{\perp}$ denotes the complementary set of $S$. Next, to model the noise we add a random noise matrix $\boldsymbol{Z}^{\bar{s}}$.

$$
\boldsymbol{Z}_{i, j}^{\bar{s}}= \begin{cases}\boldsymbol{Z}_{i, j} & \text { if }(i, j) \in S^{\perp} \\ 0 & \text { otherwise. }\end{cases}
$$

We do not assume a prior distribution on $\boldsymbol{Z}$, and the main theorem is stated for any general noise matrix $\boldsymbol{Z}$, deterministic or random.

Finally, to model the random missing entries, we assume that each entry of $\boldsymbol{D}^{\bar{s}}+t_{0} c_{0} \boldsymbol{A}^{\bar{s}}+\boldsymbol{Z}^{\bar{s}}$ is sampled with probability $p<1$. Let $E \subseteq[n] \times[n]$ denote the subset of indices which are not erased randomly. Then a projection $\mathcal{P}_{E}: \mathbb{R}^{n \times n} \rightarrow \mathbb{R}^{n \times n}$ is defined as

$$
\mathcal{P}_{E}(\boldsymbol{M})_{i, j}= \begin{cases}\boldsymbol{M}_{i, j} & \text { if }(i, j) \in E, \\ 0 & \text { otherwise. }\end{cases}
$$

We denote the observed measurement matrix by

$$
\boldsymbol{N}^{E}=\mathcal{P}_{E}\left(\boldsymbol{D}^{\bar{s}}+d_{0} \boldsymbol{A}^{\bar{s}}+\boldsymbol{Z}^{\bar{s}}\right) .
$$

Notice that the matrix $\boldsymbol{N}^{E}$ has the same shape as $\boldsymbol{T}_{\text {inc }}$ shown schematically in Fig. 5. Now we can state the goal of our calibration problem:

Given the observed matrix $N^{E}$ and the missing indices $S \cup$ $E^{\perp}$, we want to estimate a matrix $\widehat{D}$ which is close to the correct distance matrix $\boldsymbol{D}$. Then by using $\widehat{D}$ we would like to estimate the sensor positions.

In order to achieve this goal, there are two obstacles we need to overcome. First, we need to estimate the missing entries of $\boldsymbol{N}^{E}$ and second, we want to find the sensor positions given approximate pairwise distances. The former is done by employing the matrix completion algorithm OptSpace [16] and the latter by using the classic multidimensional scaling method MDSLocalize [4].

\section{A. Calibration Error Measure}

Suppose $n$ sensors lie in an $\eta$ dimensional space. The matrix $\boldsymbol{X} \in \mathbb{R}^{n \times \eta}$ represents the position matrix of the sensors. As this calibration process is anchor-free (there are no sensors with a fixed known position), the estimated position matrix $\widehat{\boldsymbol{X}}$ will be an instance of infinitely many valid solutions. In other words, distance matrices are invariant under rigid transformations (combination of translation, rotation and reflection). In order to compare $\boldsymbol{X}$ and $\widehat{\boldsymbol{X}}$, we need to define a proper measure. 
Let $\boldsymbol{L}$ be an $n \times n$ symmetric matrix such that

$$
\boldsymbol{L}=\mathbb{\rrbracket}_{n}-(1 / n) \mathbb{1}_{n} \mathbb{1}_{n}^{T},
$$

where $\mathbb{1}_{n} \in \mathbb{R}^{n}$ is the all ones vector and $\mathbb{1}_{n}$ is the $n \times n$ identity matrix. We define the distance between $\boldsymbol{X}$ and $\widehat{\boldsymbol{X}}$ as

$$
d(\boldsymbol{X}, \widehat{\boldsymbol{X}})=\frac{1}{n}\left\|\boldsymbol{L} \boldsymbol{X} \boldsymbol{X}^{T} \boldsymbol{L}-\boldsymbol{L} \widehat{\boldsymbol{X}} \widehat{\boldsymbol{X}}^{T} \boldsymbol{L}\right\|_{F}
$$

where $\|\cdot\|_{F}$ denotes the Frobenius norm.

According to [4], [12], [25], this distance is invariant to rigid transformation of $\boldsymbol{X}$ and $\widehat{\boldsymbol{X}}$. Furthermore, $d(\boldsymbol{X}, \widehat{\boldsymbol{X}})=0$ implies that $\boldsymbol{X}$ and $\widehat{\boldsymbol{X}}$ are equal up to a rigid transformation. We later state our theoretical results in terms of the distance defined in (5).

\section{MAin Results}

We mentioned earlier that the OptSpace algorithm is not directly applicable to the squared distance matrix because of the unknown delay. Since $\boldsymbol{A}$ in (2) is a full rank matrix, the matrix $\widetilde{\boldsymbol{D}} \odot \widetilde{\boldsymbol{D}}=\left[\tilde{d}_{i, j}^{2}\right]$ no longer has rank four. Moreover, as the measurements are noisy, one cannot hope for estimating the exact value for $d_{0}$. Therefore, in the following we will provide error bounds on the reconstruction of the positions assuming that the time delay (equivalently $d_{0}$ ) is known. Afterwards, a heuristic method is proposed to estimate the value of $d_{0}$.

Theorem 1: Assume $n$ sensors are distributed independently and uniformly at random on a circular ring of width $a$ with central radius $r_{0}$ as in Fig. 4. The resulting distance matrix $D$ is corrupted by structured missing entries $D^{s}$ and measurement noise $\boldsymbol{Z}^{\bar{s}}$. Further, the entries are missing randomly with probability $p$. Let $N^{E}=\mathcal{P}_{E}\left(\boldsymbol{D}-\boldsymbol{D}^{s}+\boldsymbol{Z}^{\bar{s}}\right)$ denote the observed matrix. Define $\overline{\boldsymbol{D}}$ as the squared distance matrix. Assume $\delta_{n}=\delta r_{0} \sqrt{\log n / n}$. Then, there exist constants $C_{1}$ and $C_{2}$, such that the output of OptSpace $\widehat{\bar{D}}$ achieves

$$
\frac{1}{n}\|\overline{\boldsymbol{D}}-\widehat{\overline{\boldsymbol{D}}}\|_{F} \leq C_{1}\left(\sqrt{\frac{\log n}{n}}\right)^{3}+C_{2} \frac{\left\|\mathcal{P}_{E}\left(\boldsymbol{Y}^{\bar{s}}\right)\right\|_{2}}{p n},
$$

with probability larger than $1-n^{-3}$, provided that the right hand side is less than $\sigma_{4}(\overline{\boldsymbol{D}}) / n$. We have $\boldsymbol{Y}_{i, j}^{\bar{s}}=\boldsymbol{Z}_{i, j}^{\bar{s}^{2}}+2 \boldsymbol{Z}_{i, j}^{\bar{s}} \boldsymbol{D}_{i, j}^{\bar{s}}$.

The above theorem, in great generality, holds for any noise matrix $\boldsymbol{Z}$, deterministic or random. The above guarantees only hold up to numerical constants. To evaluate the performance of this approach in practice we provide simulation results in Section VI.

Theorem 2: Applying multidimensional scaling algorithm on $\widehat{\bar{D}}$, the error on the resulting coordinates will be bounded as follows

$$
d(\boldsymbol{X}, \widehat{\boldsymbol{X}}) \leq C_{1}\left(\sqrt{\frac{\log n}{n}}\right)^{3}+C_{2} \frac{\left\|\mathcal{P}_{E}\left(\boldsymbol{Y}^{\bar{s}}\right)\right\|_{2}}{p n},
$$

with probability larger than $1-1 / n^{3}$. (The proof is given in Appendix B).
Finally we can use a heuristic algorithm to estimate the unknown value of $d_{0}$. It simply checks for which value of $d_{0}$, the reconstructed positions produce a distance matrix closest to the recorded measurements when the effect of $d_{0}$ is removed. In fact, this algorithm guarantees that after removing the effect of the time delay, we have found the best rank 4 approximation of the distance squared matrix. In other words, if we remove exactly the mismatch $d_{0}$, we will have an incomplete version of a rank 4 matrix and after reconstruction, the measured values will be closest to the reconstructed ones.

\section{A. Computational Complexity}

Note that the calibration phase can be performed in the resting stage of the device; it does not introduce any difficulties or extra procedures to the functionalities of the device. It is also easy to compute the complexity of the calibration phase. We first need to measure the time of flights. This step is also performed in the actual ultrasound tomography and does not add computational complexity compared to real tomography. Then, we need to complete the distance matrix. The complexity of this step is $O(|E| \log n)$, where $|E|$ is the number of observed entries and $n$ is the number of sensors [6]. After the distance matrix is complete, one needs to perform the MDSLocalize algorithm. This step requires a singular value decomposition which has a complexity of $O\left(n^{3}\right)$. There are algorithms which can reduce this to $O\left(n^{2}\right)$ in special cases [26].

\section{PROOF OF THEOREM 1}

In order to prove our main result, we apply Theorem 1.2 of [6] to the rank-4 matrix $\bar{D}$ and the observed matrix $\boldsymbol{N}^{E}=\mathcal{P}_{E}(\bar{D}-$ $\left.\bar{D}^{s}+\boldsymbol{Z}^{\bar{s}}\right)$.

First, we provide the definition of a crucial property of $\overline{\boldsymbol{D}}$ which is called incoherence. Following the definition in [6], a rank-4 symmetric matrix $\overline{\boldsymbol{D}} \in \mathbb{R}^{n \times n}$ is said to be $\mu$-incoherent if the following conditions hold. Let $U \Sigma U^{T}$ be the singular value decomposition of $\overline{\boldsymbol{D}}$.

A0. For all $i \in[n]$, we have $\sum_{k=1}^{4} U_{i, k}^{2} \leq 4 \mu / n$.

A1. For all $i \in[n], j \in[n]$, we have $\left|\overline{\boldsymbol{D}}_{i, j} / \sigma_{1}(\overline{\boldsymbol{D}})\right| \leq$ $\sqrt{4} \mu / n$.

The extra $1 / n$ terms in the right hand side are due to the fact that, in this paper, we assume that the singular vectors are normalized to unit norm, whereas in [6] the singular vectors are normalized to have norm $\sqrt{n}$.

Theorem 1.2 of [6] states that if a rank-4 matrix $\bar{D}$ is $\mu$-incoherent then the following is true with probability at least $1-$ $1 / n^{3}$. Let $\sigma_{i}(\overline{\boldsymbol{D}})$ be the $i$ th singular value of $\overline{\boldsymbol{D}}$ and $\kappa(\overline{\boldsymbol{D}})=$ $\sigma_{1}(\overline{\boldsymbol{D}}) / \sigma_{4}(\overline{\boldsymbol{D}})$ be the condition number of $\overline{\boldsymbol{D}}$. Also, let $\widehat{\overline{\boldsymbol{D}}}$ denote the estimation returned by OptSpace with input $N^{E}=$ $\mathcal{P}_{E}\left(\overline{\boldsymbol{D}}-\overline{\boldsymbol{D}}^{s}+\boldsymbol{Y}^{\bar{s}}\right)$. Then, there exists numerical constants $C_{1}$ and $C_{2}$ such that

$$
\frac{1}{n}\|\overline{\boldsymbol{D}}-\widehat{\overline{\boldsymbol{D}}}\|_{F} \leq C_{1} \frac{\left\|\mathcal{P}_{E}\left(\overline{\boldsymbol{D}}^{s}\right)\right\|_{2}+\left\|\mathcal{P}_{E}\left(\mathbf{Y}^{\bar{s}}\right)\right\|_{2}}{p n},
$$

provided that

$$
n p \geq C_{2} \mu^{2} \kappa(\overline{\boldsymbol{D}})^{6} \log n,
$$


and

$$
C_{1} \frac{\left\|\mathcal{P}_{E}\left(\overline{\boldsymbol{D}}^{s}\right)\right\|_{2}+\left\|\mathcal{P}_{E}\left(\boldsymbol{Y}^{\bar{s}}\right)\right\|_{2}}{p n} \leq \frac{\sigma_{4}(\overline{\boldsymbol{D}})}{n} .
$$

First, using Lemma 2, we show that the bound in (8) gives the desired bound in the theorem. Then, it is enough to show that there exists a numerical constant $N$ such that the conditions in (9) and(10) are satisfied with high probability for $n \geq N$.

Lemma 2: In the model defined in the previous section, $n$ sensors are distributed independently and uniformly at random on a circular ring of width $a$ with central radius $r_{0}$. Then, with probability larger than $1-n^{-3}$, there exists a constant $c$ such that

$$
\left\|\mathcal{P}_{E}\left(\overline{\boldsymbol{D}}^{s}\right)\right\|_{2} \leq c \delta^{3}\left(r_{0}+a\right)^{2}\left(\sqrt{\frac{\log n}{n}}\right)^{3} p n
$$

where $\mathcal{P}_{E}(\cdot)$ and $\bar{D}^{s}$ are defined as in (3). The proof of this lemma can be found in Appendix $\mathrm{C}$

Now, to show that (9) holds with high probability for $n \geq$ $C \log n / p$ for some constant $C$, we show that $\kappa \leq f_{\kappa}\left(r_{0}, \bar{a}\right)$ and $\mu \leq f_{\mu}\left(r_{0}, a\right)$ with high probability, where $f_{\kappa}$ and $f_{\mu}$ are independent of $n$. Recall that $\kappa(\overline{\boldsymbol{D}})=\sigma_{1}(\overline{\boldsymbol{D}}) / \sigma_{4}(\overline{\boldsymbol{D}})$. We have

$$
\begin{aligned}
\overline{\boldsymbol{D}}_{i, j} & =\left\|\boldsymbol{x}_{i}\right\|^{2}+\left\|\boldsymbol{x}_{j}\right\|^{2}-2 \boldsymbol{x}_{i}^{T} \boldsymbol{x}_{j} \\
& =\left(r_{0}+\rho_{i}\right)^{2}+\left(r_{0}+\rho_{j}\right)^{2}-2 \mathbf{x}_{i}^{T} \boldsymbol{x}_{j} \\
& =2 r_{0}^{2}+\left(2 r_{0} \rho_{i}+\rho_{i}^{2}\right)+\left(2 r_{0} \rho_{j}+\rho_{j}^{2}\right)-2 \boldsymbol{x}_{i}^{T} \boldsymbol{x}_{j}
\end{aligned}
$$

where $\rho_{i}$ is distributed in such a way that we have uniform distribution over the circular band. Thus, one can show that

$$
\begin{aligned}
\overline{\boldsymbol{D}} & =\boldsymbol{A} \boldsymbol{S} \boldsymbol{A}^{T}, \\
\boldsymbol{A} & =\left[\begin{array}{cccc}
r_{0} & x_{1,1} & x_{1,2} & 2 r_{0} \rho_{1}+\rho_{1}^{2} \\
\vdots & \vdots & \vdots & \vdots \\
r_{0} & x_{n, 2} & x_{n, 2} & 2 r_{0} \rho_{n}+\rho_{n}^{2}
\end{array}\right], \\
\boldsymbol{S} & =\left[\begin{array}{cccc}
2 & 0 & 0 & \frac{1}{r_{0}} \\
0 & -2 & 0 & 0 \\
0 & 0 & -2 & 0 \\
\frac{1}{r_{0}} & 0 & 0 & 0
\end{array}\right] .
\end{aligned}
$$

One can write $S$ as

$$
\begin{aligned}
& \boldsymbol{S}=\boldsymbol{U} \boldsymbol{\Lambda} \boldsymbol{U}^{-1}, \\
& \boldsymbol{\Lambda}=\operatorname{diag}\left(-2,-2, \frac{r_{0}+\sqrt{1+r_{0}^{2}}}{r_{0}}, \frac{r_{0}-\sqrt{1+r_{0}^{2}}}{r_{0}}\right) .
\end{aligned}
$$

It follows that $\sigma_{1}(\overline{\boldsymbol{D}}) \leq \frac{r_{0}+\sqrt{1+r_{0}^{2}}}{r_{0}} \sigma_{1}\left(\boldsymbol{A} \boldsymbol{A}^{T}\right)$ and $\sigma_{4}(\overline{\boldsymbol{D}}) \geq \min \left(2, \frac{\sqrt{1+r_{0}^{2}}-r_{0}}{r_{0}}\right) \sigma_{4}\left(\boldsymbol{A} \boldsymbol{A}^{T}\right)$. We can compute the expectation of this matrix over the distribution of node positions. Having uniform distribution of the sensors over the circular ring, we have for the probability distribution of $\rho$ :

$$
p_{\rho}(\rho)=\frac{r_{0}+\rho}{r_{0} a}, \text { for }-\frac{a}{2} \leq \rho \leq \frac{a}{2} .
$$

Thus, the expectation of the matrix $\boldsymbol{A}^{T} \boldsymbol{A}$ is easily computed as

$\mathbb{E}\left[\boldsymbol{A}^{T} \boldsymbol{A}\right]=\left[\begin{array}{cccc}n r_{0}^{2} & 0 & 0 & n r_{0} \frac{a^{2}}{4} \\ 0 & \frac{n}{2}\left(r_{0}^{2}+\frac{a^{2}}{4}\right) & 0 & 0 \\ 0 & 0 & \frac{n}{2}\left(r_{0}^{2}+\frac{a^{2}}{4}\right) & 0 \\ n r_{0} \frac{a^{2}}{4} & 0 & 0 & n\left(\frac{a^{2}}{16}+\frac{r_{0}^{2} a^{2}}{3}\right)\end{array}\right]$.

Let the largest and smallest singular values of $\mathbb{E}\left[\boldsymbol{A}^{T} \boldsymbol{A}\right]$ to be $n \sigma_{\max }\left(r_{0}, a\right)$ and $n \sigma_{\min }\left(r_{0}, a\right)$. Using the fact that $\sigma_{i}(\cdot)$ is a Lipschitz continuous function of its arguments, together with the Chernoff bound for large deviation of sums of i.i.d. random variables, we get

$$
\begin{aligned}
& \mathbb{P}\left(\sigma_{1}\left(\boldsymbol{A} \boldsymbol{A}^{T}\right)>2 n \sigma_{\max }\left(r_{0}, a\right)\right) \leq e^{-C n} \\
& \mathbb{P}\left(\sigma_{1}\left(\boldsymbol{A} \boldsymbol{A}^{T}\right)<(1 / 2) n \sigma_{\max }\left(r_{0}, a\right)\right) \leq e^{-C n} \\
& \mathbb{P}\left(\sigma_{4}\left(\boldsymbol{A} \boldsymbol{A}^{T}\right)<(1 / 2) n \sigma_{\min }\left(r_{0}, a\right)\right) \leq e^{-C n},
\end{aligned}
$$

for some constant $C$. Hence, with high probability, $\kappa(\overline{\boldsymbol{D}}) \leq$ $\frac{4 \sigma_{\max }\left(r_{0}, a\right)}{\sigma_{\min }\left(r_{0}, a\right)}=f_{\kappa}\left(r_{0}, a\right)$.

Now to bound $\mu$, note that with probability 1 the columns of $\boldsymbol{A}$ are linearly independent. Therefore, there exists a matrix $\boldsymbol{B} \in \mathbb{R}^{r \times r}$ such that $\boldsymbol{A}=\boldsymbol{V} \boldsymbol{B}^{T}$ with $\boldsymbol{V}^{T} \boldsymbol{V}=\rrbracket$. The SVD of $\overline{\boldsymbol{D}}$ then reads $\overline{\boldsymbol{D}}=\boldsymbol{U} \Sigma \boldsymbol{U}^{T}$ with $\Sigma=\boldsymbol{Q}^{T} \boldsymbol{B}^{T} \boldsymbol{S} \boldsymbol{B} \boldsymbol{Q}$ and $\boldsymbol{U}=\boldsymbol{V} \boldsymbol{Q}$ for some orthogonal matrix $\boldsymbol{Q}$. To show incoherence property A0, we need to show that, for all $i \in[n]$,

$$
\left\|\boldsymbol{V}_{i}\right\|^{2} \leq \frac{4 \mu}{n}
$$

Since $\boldsymbol{V}_{i}=\boldsymbol{B}^{-1} \boldsymbol{A}_{i}$, we have $\left\|\boldsymbol{V}_{i}\right\|^{2} \leq \sigma_{4}(\boldsymbol{B})^{-2}\left\|\boldsymbol{A}_{i}\right\|^{2} \leq$ $\sigma_{4}(\boldsymbol{A})^{-2}\left\|\boldsymbol{A}_{i}\right\|^{2}$. Combined with $\left\|\boldsymbol{A}_{i}\right\|^{2}=r_{0}^{2}+\left(r_{0}+\rho_{i}\right)^{2}+$ $\left(2 r_{0} \rho_{i}+\rho_{i}^{2}\right)^{2} \leq r_{0}^{2}+\left(r_{0}+a\right)^{2}+\left(2 r_{0} a+a^{2}\right)^{2}$ and (13), we have

$$
\left\|\boldsymbol{U}_{i}\right\|^{2} \leq \frac{f_{\mu}\left(r_{0}, a\right)}{n}
$$

with high probability, where $f_{\mu}\left(r_{0}, a\right)=2\left(r_{0}^{2}+\left(r_{0}+a\right)^{2}+\right.$ $\left.\left(2 r_{0} a+a^{2}\right)^{2}\right)$.

To show incoherence property $\mathbf{A} \mathbf{1}$, we use $\left|\overline{\boldsymbol{D}}_{i j}\right| \leq\left(2 r_{0}+a\right)^{2}$ and $\sigma_{1}(\overline{\boldsymbol{D}}) \geq \frac{1}{4} n \sigma_{\min }\left(r_{0}, a\right) \min \left(2, \frac{\sqrt{1+r_{0}^{2}}-r_{0}}{r_{0}}\right)$ from (12). Then,

$$
\frac{\left|\overline{\boldsymbol{D}}_{i j}\right|}{\sigma_{1}(\overline{\boldsymbol{D}})} \leq \frac{g\left(r_{0}, a\right)}{n}
$$

with high probability, where $g\left(r_{0}, a\right)=$ $\max \left(2, \frac{4 r_{0}}{\sqrt{1+r_{0}^{2}}-r_{0}}\right)\left(2 r_{0}+a\right)^{2} / \sigma_{\min }\left(r_{0}, a\right)$. Combining (14) and(15), we see that the incoherence property is satisfied, with high probability.

Further, (10) holds, with high probability, if the right-hand side of(6) is less than $C_{3} \frac{\sqrt{1+r_{0}^{2}}+r_{0}}{r_{0}} \sigma_{\max }\left(r_{0}, a\right)$, since $\sigma_{4}(\overline{\boldsymbol{D}}) \leq$ $\frac{1}{2} n \frac{\sqrt{1+r_{0}^{2}}+r_{0}}{r_{0}} \sigma_{\max }\left(r_{0}, a\right)$. This finishes the proof of Theorem 1 . 


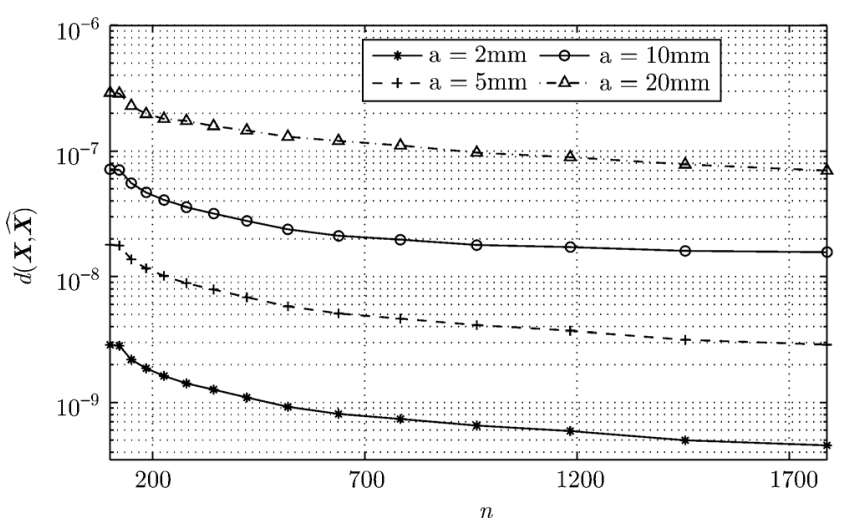

Fig. 7. Error in position estimation in noiseless case for different values of $a$. As $n$ increases, the reconstruction error tends to zero. The estimation error increases for larger values of $a$, which confirms the results of Lemma 1.

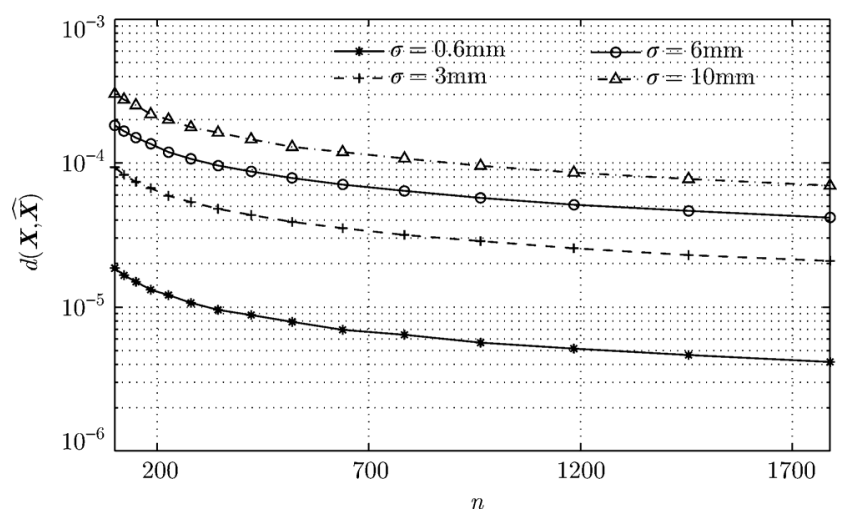

Fig. 8. Error in position estimation for the case with centered white Gaussian noise of different standard deviations, $\sigma$.

\section{Simulation Results}

Three sets of experiments are done. First, the distance matrix is assumed noiseless and the value of $d_{0}$ is set to zero. The position estimation error is derived for different values of $n$ and the ring width $a$. The value of $r_{0}$ is set to $10 \mathrm{~cm}$, on average 5 percent of entries are missing randomly, and $\delta$ in Theorem 1 is assumed to be 1 . For each value of $a$ and $n$, the experiment is repeated 10 times, and the average is taken. The results are reported in Fig. 7. As expected from Theorem 2, the greneral trend in all curves is that the error decreases as $n$ grows. Moreover, the larger $a$ is, the bigger is the reconstruction error, which is also coherent with the results of Theorem 2.

To examine the stability of the estimation algorithm under noise, under the same settings as the previous experiment, we added to each entry of the distance matrix $\boldsymbol{D}$ a centred white Gaussian noise of different standard deviations. For each $n$ and standard deviation of noise, the experiments are repeated 10 times and the average is taken. The results are depicted in Fig. ${ }^{1}$ . As the variance of the noise increases, the position estimation error grows, but in general the error decreases for larger $n$.

As we discussed in Section I-B, one might treat the calibration problem as a special case of the sensor localization problem. In order to compare the performance of sensor localization methods based on local information with the proposed methods, a set of simulations are performed. We compared the

${ }^{1}$ There has been a slight mislabeling in the earlier version of this paper in [1] which is corrected in this paper.

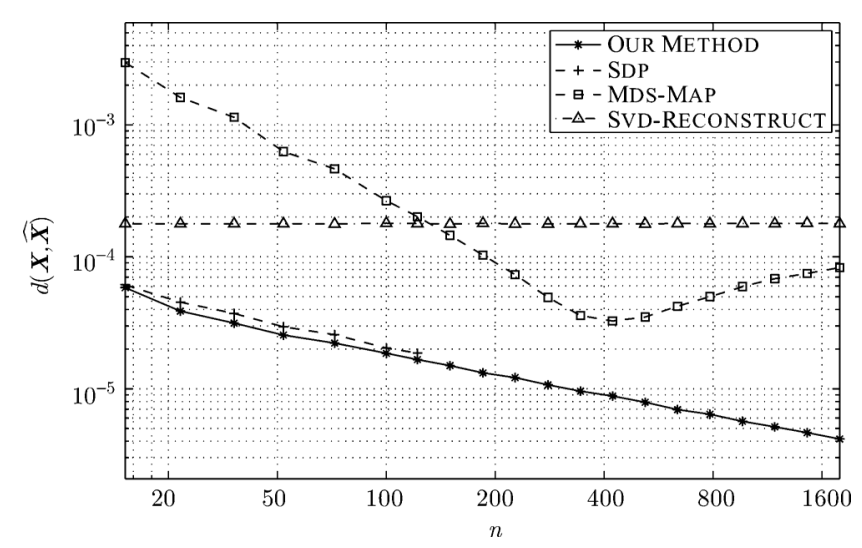

Fig. 9. Error in position estimation versus the number of sensors for different methods.

localization results of our method to the ones of Mds-Map[12], Sdp-based [13] and also Svd-Reconstruct[4]. The position reconstruction error (defined in (5)) versus the number of sensors, $n$ for the methods is reported in Fig. 9.

For the simulations, we set the values of $a$ to $1 \mathrm{~cm}, \delta$ to $1, r_{0}$ to $10 \mathrm{~cm}, t_{0}$ to zero, and the percentage of the random missing entries to 5 . The distance measurements were corrupted with a white Gaussian noise of standard deviation $0.6 \mathrm{~mm}$. For each method and each $n$, the experiment is performed 10 times for different positions and different noises, and the average error is taken. For the Sdp-based method, we used the algorithm presented in [13] and the code published by the same authors. For Mds-Map, we have estimated the shortest paths using Johnson's algorithm [24]. Finally for Svd-Reconstruct, we used the algorithm in [4]. In order to adapt the measurements with the assumptions of the method, we assumed that $p_{i j}=1-0.05=$ 0.95 for the measured points (note that 0.05 is on average the probability of having a random missing entry) and $\gamma_{i j}=0$.

As the results in Fig. 9 suggest, Mds-Map and Svd-Reconstruct perform poorer compared to the other two methods. The poor performance of Mds-Map is for the fact that it highly relies on the presence of local distance information, whereas in our case, these measurements are in fact missing. Also note that as the simulation results show, the estimation error might increase as $n$ grows.

For Svd-Reconstruct, the unrealistic assumption that all the sensors have a non-zero probability of being connected causes the bad results of the method. In our case, the probability that the close-by sensors are connected is zero because of the structured missing entries. In fact, since $p_{i j}$ is high, one could see this method as simply applying the classical MDS on the incomplete distance matrix.

In contrast to the two aforementioned algorithms, the Sdpbased method performs very well for estimating the sensor positions and the reconstruction error is very close to the one of the proposed method. This is due to the fact that this method does not directly rely on the local distance information. As the number of sensors grows, however, the number of constraints for the semi-definite program grow, which causes the algorithm intractable for $n$ larger than 150 in our case. The same limitation is also reported by the authors of the method. 


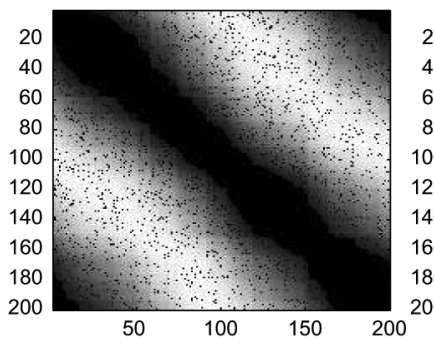

(a)

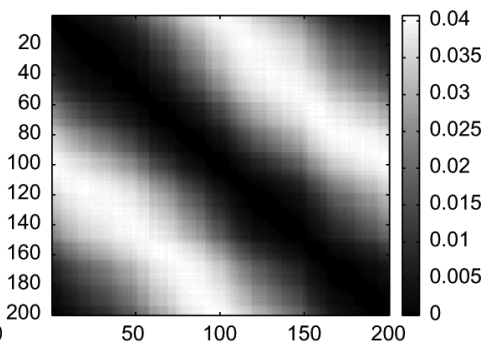

(b)
Fig. 10. Input and output of OptSpace algorithm. (a) The incomplete distance squared matrix $\bar{D}$, with 5 percent of entries randomly missing, $t_{0}=10 \mu \mathrm{s}$ and $\delta_{n}=3 \mathrm{~cm}$. (b) The completed matrix with estimated $t_{0}=10 \mu \mathrm{s}$.

In summary, taking the computational cost and reconstruction accuracy of the algorithms into account, the proposed method performs significantly better.

Moreover, to show the importance of calibration in an ultrasound scanning device, a simple simulation is also performed. Early diagnosis of breast cancer requires sub-millimeter precision in the imaging device. This implicitly poses restrictions on the calibration properties of the measuring device. If the ToF measurements correspond to the exact positions of sensors without time delay $t_{0}$, reconstruction of water will lead to a homogeneous region with values equal to the water sound speed, whereas wrong assumption on the sensor positions and $t_{0}$ causes the inverse method to give incorrect values as the sound speed to compensate the effect of position mismatch.

In a simple experiment, we simulated the reconstruction of water sound speed $\left(c_{0}=1500\right)$ using the ToF measurements. 200 sensors are distributed around a circle with radius $r_{0}=$ $10 \mathrm{~cm}$, and they deviate at most $5 \mathrm{~mm}$ from the circumference and the ToF measurements are added by $t_{0}=10 \mu \mathrm{s}$. The incomplete distance matrix is shown in Fig. 10(a). The value for $t_{0}$ is estimated as $10 \mu \mathrm{s}$ using the proposed heuristic method, which is exactly as set in the simulation. The output of OptSpace algorithm is the completed $\bar{D}$ matrix which is shown in Fig. 10(b).

Using the completed distance matrix and the MDS method, the positions are reconstructed and fed to an inverse tomography algorithm to reconstruct water sound speed. The results of the reconstruction are shown in Fig. 11. In Fig. 11(a), the ToF matrix is not complete, it contains the time delay $t_{0}$, and the positions are not calibrated. The dark gray ring is caused by the non-zero time delay in the ToF measurements. In Fig. 11(b), the time mismatch is resolved using the proposed algorithm, but the sensor positions are not calibrated and the ToF matrix is still not complete. This figure shows clearly that finding the unknown time delay improves significantly the reconstruction image. Fig. 11(c), shows the reconstructed medium when the ToF matrix is completed and time mismatch is removed, but the sensor positions are not yet calibrated. From this figure, it is confirmed that accurate time-of-flights are necessary but not sufficient to have a good reconstruction of the inclosed object. Finally, Fig. 11(d) shows the reconstruction when the positions are also calibrated. Notice the change in the dynamic range for the last case.

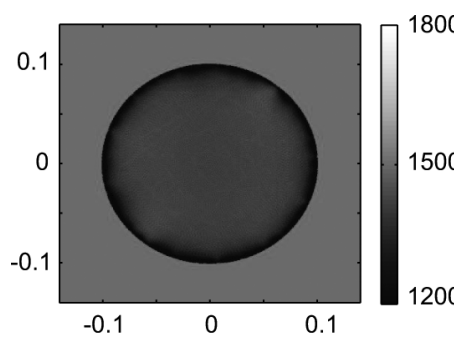

(a)

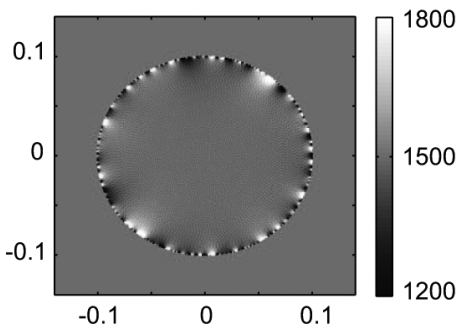

(c)

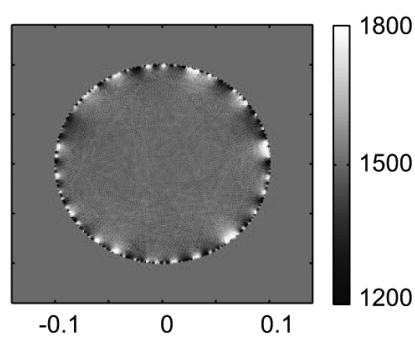

(b)

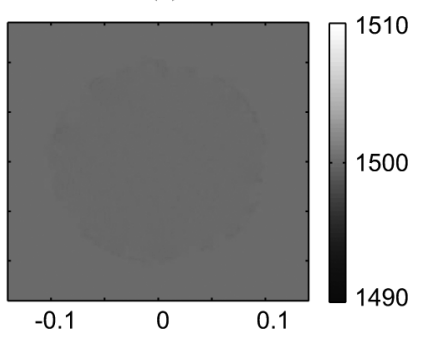

(d)
Fig. 11. Results of the inversion procedure for finding the sound speed inside the ring with only water inside. (a) Reconstruction when no calibration in performed. (b) Same after $t_{0}$ is removed from the ToF matrix, but the matrix is still incomplete and the positions are not calibrated. (c) Reconstruction when the matrix is also completed, but the positions are not yet calibrated. (d) Reconstruction with completed ToF matrix and calibrated positions.

\section{CONCLUSION}

In this work we introduced a theoretical framework for calibration in circular ultrasound tomography devices. We proposed a novel calibration algorithm for which we provided theoretical bounds on the performance. We also tested our method through exhaustive simulations to demonstrate its functionality in practice. We compared the algorithm with the state-of-the-art centralized sensor localization methods and showed that our method outperforms those in estimating the correct sensor positions.

\section{APPENDIX A \\ PROOF OF LEMMA 1}

The proof for the general case where the sensors are not on a circle is provided in [4]. In the circular case however, we have $\overline{\boldsymbol{D}}_{i, j}=\left\|\boldsymbol{x}_{i}\right\|^{2}+\left\|\mathbf{x}_{j}\right\|^{2}-2 \boldsymbol{x}_{i}^{T} \boldsymbol{x}_{j}=2 r^{2}-2 \boldsymbol{x}_{i}^{T} \mathbf{x}_{j}$, where $r$ is the circle radius. Thus, the squared distance matrix is decomposable to

$$
\begin{aligned}
\bar{D} & =\boldsymbol{V} \boldsymbol{\Sigma} \boldsymbol{V}^{T}, \\
\boldsymbol{V} & =\left[\begin{array}{ccc}
r & x_{1,1} & x_{1,2} \\
\vdots & \vdots & \vdots \\
r & x_{n, 1} & x_{n, 2}
\end{array}\right], \quad \boldsymbol{\Sigma}=\left[\begin{array}{ccc}
2 & 0 & 0 \\
0 & -2 & 0 \\
0 & 0 & -2
\end{array}\right] .
\end{aligned}
$$




\section{APPENDIX B \\ PROOF OF COROLLARY 2}

Note that in general $\left(\boldsymbol{L} \boldsymbol{X} \boldsymbol{X}^{T} \boldsymbol{L}-\boldsymbol{L} \widehat{\boldsymbol{X}} \widehat{\mathbf{X}}^{T} \boldsymbol{L}\right)$ has rank at most $2 d$ where $d$ is the dimension of the space in which sensors are placed (in our case $d=2$ ). Therefore,

$$
\left\|\boldsymbol{L} \boldsymbol{X} \boldsymbol{X}^{T} \boldsymbol{L}-\boldsymbol{L} \widehat{\mathbf{X}} \widehat{\boldsymbol{X}}^{T} \boldsymbol{L}\right\|_{F} \leq \sqrt{2 d}\left\|\boldsymbol{L} \boldsymbol{X} \boldsymbol{X}^{T} \boldsymbol{L}-\boldsymbol{L} \widehat{\boldsymbol{X}} \widehat{\mathbf{X}}^{T} \boldsymbol{L}\right\|_{2},
$$

where we used the fact that for any matrix $A$ of rank $r$ we have $\|A\|_{F} \leq \sqrt{r}\|A\|_{2}$. Furthermore, the spectral norm can be bounded in terms of $\overline{\boldsymbol{D}}$ and $\widehat{\bar{D}}$ as follows.

$$
\begin{aligned}
& \left\|\boldsymbol{L} \boldsymbol{X} \boldsymbol{X}^{T} \boldsymbol{L}-\boldsymbol{L} \widehat{\mathbf{X}} \widehat{\boldsymbol{X}}^{T} \boldsymbol{L}\right\|_{2} \\
& \stackrel{(a)}{\leq}\left\|\boldsymbol{L} \boldsymbol{X} \boldsymbol{X}^{T} \boldsymbol{L}-\frac{1}{2} \boldsymbol{L} \widehat{\overline{\boldsymbol{D}}} \boldsymbol{L}\right\|_{2}+\left\|\frac{1}{2} \boldsymbol{L} \widehat{\overline{\boldsymbol{D}}} \boldsymbol{L}-\widehat{\mathbf{X}} \widehat{\boldsymbol{X}}^{T}\right\|_{2} \\
& \stackrel{(b)}{\leq} \frac{1}{2}\|\boldsymbol{L}(\overline{\mathbf{D}}-\widehat{\widehat{\boldsymbol{D}}}) \boldsymbol{L}\|_{2}+\frac{1}{2}\|\boldsymbol{L}(-\overline{\boldsymbol{D}}+\widehat{\overline{\boldsymbol{D}}}) \boldsymbol{L}\|_{2},
\end{aligned}
$$

where in $(a)$, we used the triangle inequality and $\boldsymbol{L} \widehat{\boldsymbol{X}}=\widehat{\boldsymbol{X}}$. In $(b)$, we used

$$
-\frac{1}{2} \boldsymbol{L} \bar{D} \boldsymbol{L}=\boldsymbol{L} \boldsymbol{X} \mathbf{X}^{T} \boldsymbol{L},
$$

and the fact that for any matrix $A$ of rank $d$, $\left\|\frac{1}{2} \boldsymbol{L} \widehat{\bar{D}} \boldsymbol{L}-\widehat{\mathbf{X}} \widehat{\boldsymbol{X}}^{T}\right\|_{2} \leq\left\|\frac{1}{2} \boldsymbol{L} \widehat{\overline{\boldsymbol{D}}} \boldsymbol{L}-A\right\|_{2}$. In particular, by setting $A=\frac{1}{2} \boldsymbol{L} \overline{\boldsymbol{D}} \boldsymbol{L}$ the second term in (16) follows. Since $\boldsymbol{L}$ is a projection matrix we have $\|\boldsymbol{L}\|_{2}=1$. Hence, from (16) we can conclude that

$$
\left\|\boldsymbol{L} \boldsymbol{X} \boldsymbol{X}^{T} \boldsymbol{L}-\boldsymbol{L} \widehat{\mathbf{X}} \widehat{\boldsymbol{X}}^{T} \boldsymbol{L}\right\|_{2} \leq\|\widehat{\overline{\boldsymbol{D}}}-\overline{\boldsymbol{D}}\|_{2} .
$$

This immediately leads to Corollary 2 .

\section{APPENDIX C \\ PROOF OF LEMMA 2}

Note that by the definition of $\overline{\boldsymbol{D}}^{s}$, we have $\left|\mathcal{P}_{E}\left(\overline{\boldsymbol{D}}^{s}\right)_{i, j}\right| \leq \delta_{n}^{2}$ for all $i$ and $j$. Define $\boldsymbol{A}$ as

$$
\boldsymbol{A}_{i, j}= \begin{cases}1 & \text { if }(i, j) \in E \cap S \\ 0 & \text { otherwise }\end{cases}
$$

We start from a simple realtionship between an elementwise bounded matrix and its operator norm.

$$
\left\|\mathcal{P}_{E}\left(\overline{\boldsymbol{D}}^{s}\right)\right\|_{2} \leq \delta_{n}^{2} \max _{\|x\|=\|y\|=1} \sum_{i, j}\left|x_{i}\right|\left|y_{j}\right| \boldsymbol{A}_{i, j}=\delta_{n}^{2}\|\boldsymbol{A}\|_{2} .
$$

The inequlity in (17) follows from the fact that $\mathcal{P}_{E}\left(\overline{\boldsymbol{D}}^{s}\right)$ is elementwise bounded by $\delta_{n}$. We can further bound the operator norm $\|\boldsymbol{A}\|_{2}$, by applying the celebrated Gershgorin circle theorem to a symmetrized version of $\boldsymbol{A}$. Define a symmetric matix $\bar{A}$ as

$$
\overline{\boldsymbol{A}}_{i, j}= \begin{cases}1 & \text { if }(i, j) \in E \cap S \mathrm{or}(j, i) \in E \cap S, \\ 0 & \text { otherwise }\end{cases}
$$

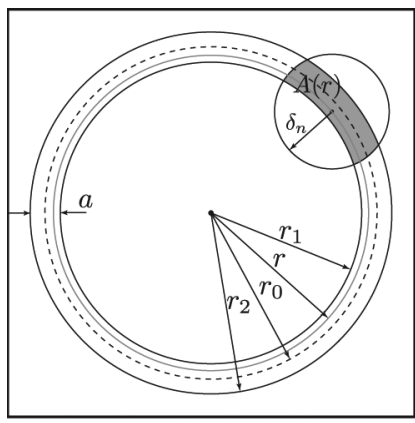

(a)

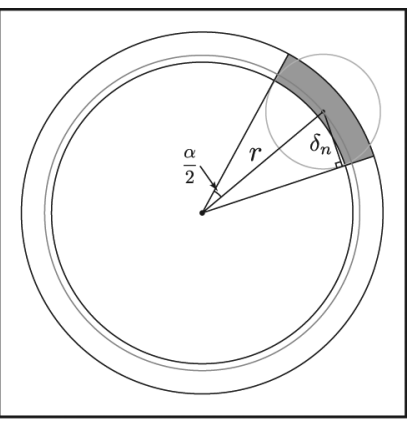

(b)
Fig. 12. (a) The process for bounding the probability of a pair of sensors to fall in $S . r_{1}=r_{0}-a / 2$ and $r_{2}=r_{0}+a / 2$. (b) Upper bound on $A(r)$. The grey area made by the tangents to the $\delta_{n}$ circle is an upper bound for $A(r)$.

Since $0 \leq \boldsymbol{A}_{i, j} \leq \overline{\boldsymbol{A}}_{i, j}$ for all $i$ and $j$, we have $\|\boldsymbol{A}\|_{2} \leq\|\overline{\boldsymbol{A}}\|_{2}$. Applying the Gershgorin circle theorem we get

$$
\|\overline{\boldsymbol{A}}\|_{2} \leq \max _{i \in[n]} \sum_{j \in[n]}\left|\bar{A}_{i, j}\right|
$$

Define random variables $\left\{Y_{1}, \ldots, Y_{n}\right\}$, where $Y_{i}$ is the number of non-zero entries in the $i$ th row of $\overline{\boldsymbol{A}}$. Then,

$$
\|\overline{\boldsymbol{A}}\|_{2} \leq \max _{i \in[n]} Y_{i}
$$

We need to show that $Y_{i}$ concentrates around its mean. Since $Y_{i}$ 's are binomial random variables, we can apply the Chernoff bound. Recall that $(i, j) \in S$ if $\left\|\boldsymbol{x}_{i}-\boldsymbol{x}_{j}\right\| \leq \delta_{n}$. By the definition of $E$, each sample is sampled with probability $p$. Then the probability that either $(i, j)$ or $(j, i)$ is in $E$ is $2 p-p^{2}$.

Each entry in the $i$ th row of $\bar{A}$ is an independent Bernoulli random variable with probability of being one equal to $q(2 p-$ $p^{2}$ ), where $q$ is the probability that a pair is in $S$. Thus, we have $\mathbb{E}\left[Y_{i}\right]=q\left(2 p-p^{2}\right) n$. In order to find the bounds on $\mathbb{E}\left[Y_{i}\right]$, we need to bound $q$. Fig. 12(a) shows the process for obtaining the bounds on $q$.

$$
q=\mathbb{P}\left\{\left|\boldsymbol{x}_{i}-\boldsymbol{x}_{j}\right| \leq \delta_{n}\right\}=\int_{r_{1}}^{r_{2}} \frac{2 \pi r}{\pi\left(r_{2}^{2}-r_{1}^{2}\right)} p_{2}(r) d r,
$$

where $p_{2}(r)=A(r) /\left(\pi\left(r_{2}^{2}-r_{1}^{2}\right)\right)$.

Upper Bound On $A(r)$ : Obviously the area $A(r)$ can be bounded by what is shown in Fig. 12(b) Thus, we will have $\sin (\alpha / 2)=\delta_{n} / r$.

Note that for $0<\alpha<\pi, \alpha / \pi \leq \sin \alpha / 2 \leq \alpha / 2$. Hence, $\alpha / \pi \leq \delta_{n} / r \leq \alpha / 2$. So,

$$
A(r) \leq \frac{\alpha}{2 \pi} \pi\left(r_{2}^{2}-r_{1}^{2}\right) \leq \frac{\delta_{n} \pi}{2 r}\left(r_{2}^{2}-r_{1}^{2}\right) .
$$

Thus

$$
\begin{aligned}
p_{2}(r) & \leq \frac{\frac{\delta_{n} \pi}{2 r}\left(r_{2}^{2}-r_{1}^{2}\right)}{\pi\left(r_{2}^{2}-r_{1}^{2}\right)}=\frac{\delta_{n}}{2 r} . \\
q & \leq \int_{r_{1}}^{r_{2}} \frac{2 \pi r}{\pi\left(r_{2}^{2}-r_{1}^{2}\right)} \cdot \frac{\delta_{n}}{2 r} d r=\frac{\delta_{n}}{r_{2}+r_{1}}=\frac{\delta_{n}}{2 r_{0}} .
\end{aligned}
$$




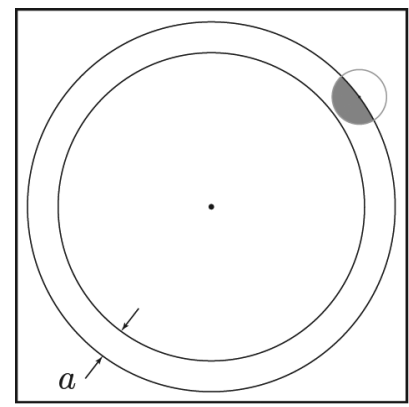

(a)

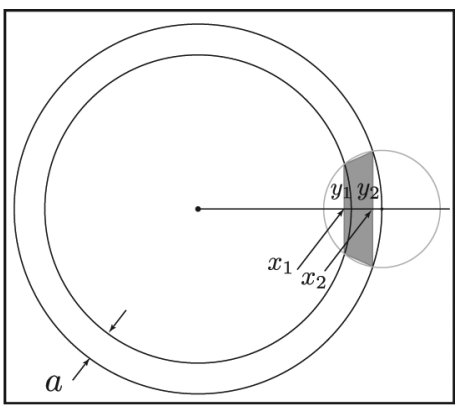

(b)
Fig. 13. Evaluation of lower bound for $A(r)$. In (a) we assume that $\delta_{n} \leq a$ whereas in (b) we take $\delta_{n}>a$. In both cases the minimum intersection is achieved when the center of $\delta_{n}$ circle is on the exterior boundary of the region.

Lower Bound on $A(r)$ : In order to find the lower bound, we consider cases:

Case $1\left(\delta_{n} \leq a\right)$ : In this case the minimum area of the intersection is achieved when the center of the circle is on the exterior boundary of the region as shown in Fig. 13(a). In this case, one can show that,

$$
A(r) \geq \frac{\pi \delta_{n}^{2}}{4} .
$$

Case $2\left(\delta_{n}>a\right)$ : In this case, the minimum area is achieved when the center of the circle is on the exterior boundary as in Fig. 13(b), where

$$
\begin{array}{ll}
x_{1}=\frac{r_{2}^{2}-\delta_{n}^{2}+r_{1}^{2}}{2 r_{2}}, & y_{1}=\frac{1}{r_{2}} \sqrt{\left(\delta_{n}^{2}-a^{2}\right)\left(4 r_{0}^{2}-\delta_{n}^{2}\right)} \\
x_{2}=\frac{r_{2}^{2}-\delta_{n}^{2}+r_{2}^{2}}{2 r_{2}}, & y_{2}=\frac{1}{r_{2}} \sqrt{\delta_{n}^{2}\left(4 r_{2}^{2}-\delta_{n}^{2}\right)} .
\end{array}
$$

Thus, we will have

$$
\begin{aligned}
A(r) & \geq \frac{y_{1}+y_{2}}{2}\left(x_{2}-x_{1}\right) \\
& \geq \frac{\sqrt{\delta_{n}^{2}\left(4 r_{2}-\delta_{n}^{2}\right)}}{2 r_{2}} \cdot \frac{r_{2}^{2}-r_{1}^{2}}{2 r_{2}} \\
& =\delta_{n} \sqrt{\left(r_{2}-\frac{1}{4} \delta_{n}^{2}\right)} \frac{r_{2}^{2}-r_{1}^{2}}{2 r_{2}^{2}} .
\end{aligned}
$$

If we assume that $r_{2} \geq \frac{1}{\sqrt{2}} \delta_{n}$, which is a reasonable assumption according to the problem statement, we will have

$$
A(r) \geq \frac{1}{2} \delta_{n}^{2} \frac{r_{2}^{2}-r_{1}^{2}}{2 r_{2}^{2}} \geq \frac{a r_{0}}{2\left(r_{0}+a\right)^{2}} \delta_{n}^{2} .
$$

Combining (17) and(18), we can find the lower bound for $A(r)$ as

$$
A(r) \geq \min \left(\frac{\pi}{4}, \frac{a r_{0}}{2\left(r_{0}+a\right)^{2}}\right) \delta_{n}^{2}=\frac{a r_{0}}{2\left(r_{0}+a\right)^{2}} \delta_{n}^{2} .
$$

Thus,

$$
\begin{aligned}
q & =\int_{r_{1}}^{r_{2}} \frac{2 \pi r}{\pi\left(r_{2}^{2}-r_{1}^{2}\right)} p_{2}(r) d r \\
& =\int_{r_{1}}^{r_{2}} \frac{2 \pi r}{\pi\left(r_{2}^{2}-r_{1}^{2}\right)} \frac{A(r)}{\pi\left(r_{2}^{2}-r_{1}^{2}\right)} d r \geq \frac{\delta_{n}^{2}}{4 \pi\left(r_{0}+a\right)^{2}} .
\end{aligned}
$$

From the above calculations, we have that $\frac{\delta_{n}^{2}}{4 \pi\left(r_{0}+a\right)^{2}} p n \leq$ $\mathbb{E}\left[Y_{i}\right] \leq \frac{1}{r_{0}} \delta_{n} p n$. Applying the Chernoff bound to $Y_{i}$, we have

$$
\mathbb{P}\left(Y_{i}>(1+\alpha) \mathbb{E}\left[Y_{i}\right]\right) \leq 2^{-(1+\alpha) \mathbb{E}\left[Y_{i}\right]} .
$$

In other words

$$
\mathbb{P}\left(Y_{i}>(1+\alpha) \frac{1}{r_{0}} \delta_{n} p n\right) \leq 2^{-(1+\alpha) \frac{\delta_{n}^{2}}{4 \pi\left(r_{0}+a\right)^{2}} p n} .
$$

Applying the union bound, we get

$$
\begin{aligned}
\mathbb{P}\left(\max _{i \in[n]} Y_{i}>(1+\alpha) \frac{1}{r_{0}} \delta_{n} p n\right) & \leq n 2^{-(1+\alpha) \frac{\delta_{n}^{2}}{4 \pi\left(r_{0}+a\right)^{2}} p n} \\
& \leq 2^{-\left((1+\alpha) \frac{\delta_{n}^{2}}{4 \pi\left(r_{0}+a\right)^{2}} p n-\log _{2} n\right)} .
\end{aligned}
$$

By the assumption that $\delta_{n} p=\Omega\left(r_{0} \sqrt{\log _{2} n / n}\right)$, there exists constants $c$ and $N$, such that $\delta_{n}^{2} p \geq c r_{0}^{2} \log _{2} n / n$, for $n \geq N$. Define a positive parameter $\beta$ such that $1+\beta=\frac{c(1+\bar{\alpha}) r_{0}^{2}}{4 \pi\left(r_{0}+a\right)^{2}}$. Then we will have

$$
\mathbb{P}\left(\max _{i \in[n]} Y_{i}>\frac{4 \pi(1+\beta)}{c r_{0}^{3}}\left(r_{0}+a\right)^{2} \delta_{n} p n\right) \leq n^{-\beta} .
$$

Finally with probability $1-n^{-\beta}$,

$$
\begin{aligned}
\left\|\mathcal{P}_{E}\left(\overline{\boldsymbol{D}}^{s}\right)\right\|_{2} & \leq \frac{4 \pi(1+\beta)}{c} \delta^{3}\left(\sqrt{\frac{\log n}{n}}\right)^{3} p n \\
& =C\left(r_{0}+a\right)^{2} \delta^{3}\left(\sqrt{\frac{\log n}{n}}\right)^{3} p n .
\end{aligned}
$$

This finishes the proof of Lemma 2.

\section{REFERENCES}

[1] R. Parhizkar, A. Karbasi, and M. Vetterli, "Calibration in circular ultrasound tomography devices," in Proc. IEEE Int. Conf. Acoust., Speech, Signal Process. , Prague, Czech Republic, May 2011, pp. 549-552.

[2] N. Duric, P. Littrup, L. Poulo, A. Babkin, R. Pevzner, E. Holsapple, O. Rama, and C. Glide, "Detection of breast cancer with ultrasound tomography: First results with the computed ultrasound risk evaluation (cure) prototype," Med. Phys., vol. 34, no. 2, 2007.

[3] I. Jovanovic, L. Sbaiz, and M. Vetterli, "Acoustic tomography for scalar and vector fields: theory and application to temperature and wind estimation," J. Atmos. Ocean. Technol., vol. 26, no. 8, pp. 1475-1492, 2009.

[4] P. Drineas, A. Javed, M. Magdon-Ismail, G. Pandurangant, R. Virrankoski, and A. Savvides, "Distance matrix reconstruction from incomplete distance information for sensor network localization," SECON, vol. 2, pp. 536-544, 2006.

[5] E. J. Candès and B. Recht, "Exact matrix completion via convex optimization," Found. Comput. Math., vol. 9, no. 6, pp. 717-772, 2009.

[6] R. H. Keshavan, A. Montanari, and S. Oh, "Matrix completion from noisy entries," Adv. Neural Inf. Process. Syst., 2009. 
[7] T. He, C. Huang, B. M. Blum, J. A. Stankovic, and T. Abdelzaher, "Range-free localization schemes for large scale sensor networks," in Proc. MobiCom,

[8] S. Čapkun, M. Hamdi, and J. Hubaux, "GPS-free positioning in mobile ad hoc networks," Cluster Comput., vol. 5, no. 2, pp. 157-167, 2002.

[9] A. Savvides, C. Han, and M. Strivastava, "Dynamic fine-grained localization in ad-hoc networks of sensors," in Proc. MobiCom, , 2001, pp. $166-179$.

[10] D. Niculescu and B. Nath, "Ad hoc positioning system (APS) using AoA," in Proc. INFOCOM, , 2003, pp. 1734-1743.

[11] C. Liu, K. Wu, and T. He, "Sensor localization with ring overlapping based on comparison of received signal strength indicator," in Proc. MASS, , 2004, pp. 516-518.

[12] Y. Shang, W. Ruml, Y. Zhang, and M. P. J. Fromherz, "Localization from mere connectivity," in Proc. MobiHoc, , 2003, pp. 201-212.

[13] P. Biswas, T. Chen Liang, K. Chuan Toh, T. Chung Wang, and Y. Ye, "Semidefinite programming approaches for sensor network localization with noisy distance measurements," IEEE Trans. Automat. Sci. Eng., vol. 3, no. 4, pp. 360-371, Oct. 2006.

[14] A. Montanari and A. Javanmard, Localization from incomplete noisy distance measurements arXiv:1103.1417v3. : , 2011.

[15] R. H. Keshavan, A. Montanari, and S. Oh, "Matrix completion from a few entries arXiv:0901.3150," IEEE Trans. Inf. Theory, vol. 56, no. 6, pp. 2980-2998, Jun. 2010.

[16] R. H. Keshavan and S. Oh, OptSpace: A gradient descent algorithm on the grassman manifold for matrix arXiv:0910.5260 completion. : , 2009.

[17] B. Recht, “A simpler approach to matrix completion," J. Mach. Learn. Res., 2011.

[18] B. Recht, W. Xu, and B. Hassibi, "Null space conditions and thresholds for rank minimization," Math. Program., vol. 127, no. 1, pp. 175-202, Mar. 2011.

[19] I. Jovanović, A. Hormati, L. Sbaiz, and M. Vetterli, "Efficient and stable acoustic tomography using sparse reconstruction methods," presented at the ICA, 2007.

[20] F. Natterer, Acoustic mammography in the time domain University of Münster, North Rhine-Westphalia, Germany, 2008. [Online]. Available: http://www.math.uni-muenster.de/num/Preprints/files/80.pdf

[21] J. Chen, Y. Huang, and J. Benesty, "Time delay estimation," in Audio Signal Processing for Next-Generation Multimedia Communication Systems. Berlin, Germany: Springer-Verlag, 2004, ch. 8, pp. $197-227$.

[22] I. Jovanović, "Inverse problems in acoustic tomography, dissertation, EPFL," Ph.D. dissertation, Lausanne, Switzerland, 2008.

[23] C. Li, L. Huang, N. Duric, H. Zhang, and C. Rowe, "An improved automatic time-of-flight picker for medical ultrasound tomography," Ultrasonics, vol. 49, no. 1, pp. 61-72, 2009.

[24] Y. Rue and S. Mylvaganam, "Performance monitoring of ultrasonic transducers with laser vibrometers," SAS, pp. 64-69, 2011.

[25] S. Oh, A. Montanari, and A. Karbasi, "Sensor network localization from local connectivity: Performance analysis for the MDS-map algorithm," ITW, pp. 1-5, 2010.

[26] T. Konda and Y. Nakamura, "A new algorithm for singular value decomposition and its parallelization," Parallel Comput., vol. 35, no. 6, pp. 331-344, 2009.

[27] D. B. Johnson, "Efficient algorithms for shortest paths in sparse networks," J. ACM, vol. 24, pp. 1-13, 1977.

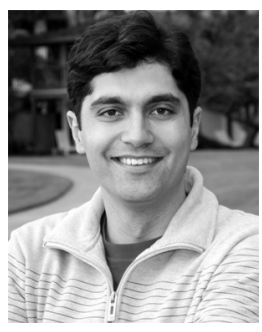

Reza Parhizkar (S'09-M'13) was born in Oroumieh, Iran in 1985. He received his B.Sc. in electrical engineering from Sharif University, Tehran in 2007 and M.Sc. in communication systems from the Swiss Federal Institute of Technology, Lausanne (EPFL) in 2009. As an intern he worked for six months in the Nokia research center in Lausanne in 2009 and for three months in Qualcomm Inc., San Diego in 2013. Since 2009, he has been a $\mathrm{Ph} . \mathrm{D}$. candidate in the audiovisual communications laboratory (LCAV) at EPFL. His work on sensor calibration for ultrasound tomography devices won the best student paper award in ICASSP 2011. His research interests include mathematical signal processing, sensor localization and inverse problems.

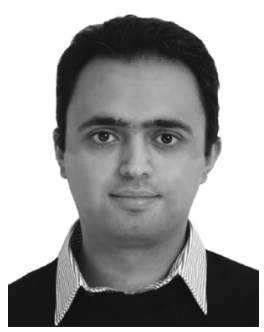

Amin Karbasi (S'11-M'12) received the B.Sc., M.Sc., and Ph.D. degrees in computer and communication sciences all from EPFL. Since March 2013, he has been a postdoctoral fellow in the computer science department at ETHZ. He was the recipient of the ICASSP 2011 best student paper award and ACM/Sigmetrics 2010 best student paper award. His research interests include graphical models, large scale networks, and high-dimensional statistics.

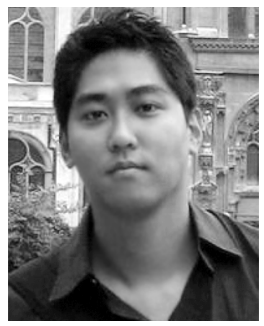

Sewoong Oh (S'07-M'11) is an Assistant Professor of Industrial and Enterprise Systems Engineering at UIUC. His research interest is in understanding how to extract meaningful information from societal data, such as aggregating opinions on social computation platforms like Mechanical Turk, making recommendations from comparisons, and finding ranking from comparisons. He received his Ph.D. from the department of Electrical Engineering at Stanford University in 2011. Following his Ph.D., he worked as a postdoctoral researcher at Laboratory for Information and Decision Systems (LIDS) at MIT. He was awarded the Kenneth C. Sevcik outstanding student paper award at the Sigmetrics 2010.

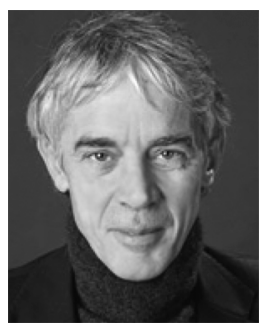

Martin Vetterli (S'86-M'86-SM'90-F'95) received the Dipl. El.- Ing. degree from Eidgenossische Technische Hochschule (ETHZ), Zurich, in 1981, the M.Sc. from Stanford University in 1982, and the Ph.D. from the EPFL, Lausanne, in 1986. After his dissertation, he was an Assistant and then Associate Professor in Electrical Engineering at Columbia University in New York. In 1993, he became an Associate and then Full Professor at the Department of Electrical Engineering and Computer Sciences at the University of California at Berkeley. In 1995, he joined the EPFL as a Full Professor and since 2013 he is also the president of the Swiss National Science Foundation. His work covers wavelet theory and applications, image and video compression, self-organized communications systems and sensor networks, as well as fast algorithms, and has led to about 150 journals papers. He is the co-author of three textbooks. His research resulted also in about two dozen patents that led to technology transfers to high-tech companies and the creation of several start-ups. His work won him numerous prizes, like best paper awards from EURASIP in 1984 and of the IEEE Signal Processing Society in 1991, 1996 and 2006, the Swiss National Latsis Prize in 1996, the SPIE Presidential award in 1999, the IEEE Signal Processing Technical Achievement Award in 2001 and the IEEE Signal Processing Society Award in 2010. He is a Fellow of IEEE, of ACM and EURASIP, was a member of the Swiss Council on Science and Technology (2000-2004), and is an ISI highly cited researcher in engineering. 